\title{
The nitroblue tetrazolium (NBT) test in renal transplantation
}

\author{
A. M. GORDON, J. D. BRIGGS, AND P. R. F. BELL \\ From the University Department of Bacteriology, Glasgow Royal Infirmary, the Renal Unit and University \\ Department of Surgery, Western Infirmary, Glasgow
}

SYNOPSIS The nitroblue tetrazolium (NBT) test has been evaluated as a means of differentiating episodes of infection and rejection in 10 cadaver kidney transplant recipients. Normal NBT values were observed during all 11 episodes of acute rejection. A significant elevation of the NBT score was encountered in six of eight episodes of infection after transplantation. A positive NBT result is useful additional evidence in favour of infection in patients in whom the differentiation of infection and rejection is proving difficult.

While renal transplantation has considerably improved the prognosis of patients with end-stage renal failure, the renal allograft recipient remains prone to postoperative episodes of infection and rejection. Infection is the main cause of death following renal transplantation (Gurland, Brunner, Dehn, Härlen, Parsons, and Scharer, 1973), while rejection is the most frequent postoperative complication (Briggs, Timbury, Paton, and Bell, 1972). A rapid and easily performed test which would assist the diagnosis of rejection and its differentiation from an infective episode would clearly be of value.

The nitroblue tetrazolium (NBT) test is now well established as a useful screening procedure for the confirmation of systemic bacterial infection and for its differentiation from non-bacterial pyrexial syndromes (Park, Fikrig, and Smithwick, 1968; Lancet, 1971; Gordon, Rowan, Brown, and Carson, 1973). It has also been suggested that this test might provide a simple means of distinguishing between episodes of rejection and infection in the immunosuppressed renal transplant recipient (Wollman, Brennan, Stenzel, David, Lewy, Ruben, and Millar, 1972). Prompted by this report we have performed serial postoperative NBT tests on individuals who have received cadaver renal transplants. We report here our experience of the value of this technique as a means of differentiating infection and rejection in these individuals in the postoperative period.

\section{Patients and Methods}

Ten patients with end-stage renal failure (six males, Received for publication 23 May 1974. four females: age range $22-42$ years) were investi gated. Seven of the patients had chronic glomerulonephritis, two had chronic pyelonephritis, and one had hereditary nephritis. All patients were main tained on regular haemodialysis before receiving a cadaver kidney. The operative technique and the postoperative management were as described by Bell, Briggs, Kyle, Dick, Calman et al (1972). Postoperative immunosuppression consisted of azathioprine and prednisolone. Azathioprine was administered once daily in a dose sufficient to maintain the total leucocyte count between 5000 and 8000 cells per $\mathrm{cmm}$. At the time of transplantation oral prednisolone was commenced in a daily dose of $200 \mathrm{mg}$, which was reduced to $15 \mathrm{mg}$ over the first three months. One gram of intravenous prednisolone was given during the transplant operation and this was repeated during acute rejection episodes as described by Bell et al (1972). The diagnosis of rejection was made mainly on the basis of biochemical criteria, the most important being a falling urine volume and a rising serum creatinine. Useful clinical guides sometimes seen included swelling of the graft, tenderness, and weight gain. Frequent bacteriological monitoring of the patients was undertaken, including cultivation of sputum, urine, and swabs from nose, throat, and wounds. If a pyrexia developed blood cultures were also performed.

Serial venous blood samples for the NBT test were collected into sequestrene (EDTA). These were obtained daily for the first four weeks following transplantation and twice weekly thereafter for up to three months. During episodes of established or presumed infection or rejection samples were obtained 
on alternate days. All specimens were processed immediately, but where delay was unavoidable they were stored at $+4^{\circ} \mathrm{C}$ (to a maximum period of 12 hours) before testing, in order to minimize spontaneous enhancement of dye reduction (Winchester, Gordon, Rowan, Lindsay, and Black, 1973). The NBT tests were performed on buffy coat preparations by the EDTA/Ficoll technique previously described by Gordon and his coworkers (1973). The preparations were examined by one experienced observer from the investigating group (A.M.G.) who remained unaware of the clinical courses of the patients after transplantation until the end of the study. As a routine 200 neutrophils with intact cytoplasmic membranes were counted under oil immersion. Cells containing reduced nitroblue tetrazolium (formazan) deposits equal to or exceeding the size of individual neutrophil lobes were considered to be NBT-positive and the percentage of NBT-positive cells was recorded as the 'NBT score'. Stippled cells and those exhibiting membrane disruption were not included in the differential count. Patients with more than $14 \%$ NBT-positive neutrophils were considered to have elevated NBT scores.

\section{Results}

\section{POSTOPERATIVE COURSE}

Three of the cadaver kidneys underwent irreversible rejection, necessitating nephrectomy and a return to maintenance dialysis after six weeks in two of the patients, and after three months in one case. One graft exhibited primary non-function and was removed six weeks after transplantation. The remaining six patients were clinically well with stable renal function nine to 12 months after receiving their transplants.

\section{COMPLICATIONS}

Table I summarizes NBT scores in relation to all postoperative complications occurring in the patients studied.

Eleven episodes of graft rejection were observed in eight patients. The diagnosis of rejection was based

\begin{tabular}{llll}
\hline Episode & \multirow{2}{*}{ Number } & \multicolumn{2}{l}{ NBT Score } \\
\cline { 3 - 4 } & & Normal & Elevated \\
\hline Rejections & 11 & 11 & 0 \\
Infections & 8 & 2 & 6 \\
Dialysis pyrexias & 5 & 5 & 0 \\
\hline
\end{tabular}

Table I NBT values in relation to episodes occurring following renal transplantation (10 patients)

on standard clinical and laboratory criteria, the main emphasis being placed on urine volume and serum creatinine (Bell et al, 1972). Three of the rejections were irreversible, leading to loss of the transplanted kidney. The remaining eight episodes were less severe and were successfully reversed by the intravenous administration of prednisolone. Eight of the 11 episodes of rejection were associated with the development of a pyrexia or a leucocytosis, thus simulating systemic bacterial infection. However, serial NBT values within the normal range were recorded during all rejections, the scores being less than $5 \%$ in six episodes and between $5 \%$ and $10 \%$ in the other five instances (table I).

Eight episodes of infection complicated the posttransplantation course of six patients. The nature of the infections and the range of NBT values encountered during each episode are recorded in table II. Multiple elevated NBT responses (as high as $54 \%$ in one instance) were found during six of the infective episodes despite the concurrent administration of immunosuppressive agents. The NBT scores within the normal range were noted in the two remaining infective episodes. These were two clinically mild respiratory infections occurring in a patient who remained apyrexial during both episodes.

Five episodes of pyrexia occurred in three patients: during haemodialysis after transplantation, but NBT tests performed during the pyrexial phases yielded normal scores (less than $10 \%$ ) in all instances.

\section{Discussion}

Infection remains the most serious complication

\begin{tabular}{|c|c|c|c|}
\hline Infection & Pathogen & $\begin{array}{l}\text { NBT Values (\%) } \\
\text { (range and mean \% score) }\end{array}$ & No. of Tests \\
\hline $\begin{array}{l}\text { Perinephric abscess } \\
\text { Perinephric abscess } \\
\text { Respiratory tract infection } \\
\text { Respiratory tract infection } \\
\text { Urinary tract infection } \\
\text { Wound infection } \\
\text { Respiratory tract infection } \\
\text { Respiratory tract infection }\end{array}$ & $\begin{array}{l}\text { None isolated } \\
\text { None isolated } \\
\text { Staph. aureus } \\
\text { Haemophilus influenzae } \\
\text { Proteus species } \\
\text { Staph. aureus } \\
\text { Haemophilus influenzae } \\
\text { Esch. coli }\end{array}$ & $\begin{array}{cl}17-54 & (32.6 \%) \\
18-34 & (26.4 \%) \\
22-38 & (28.1 \%) \\
18-22 & (19.6 \%) \\
17-23 & (20.3 \%) \\
16-40 & (30.9 \%) \\
4-8^{1} & (6 \%) \\
3-6^{1} & (4.5 \%)\end{array}$ & $\begin{array}{l}8 \\
7 \\
4 \\
4 \\
5 \\
6 \\
3 \\
4\end{array}$ \\
\hline
\end{tabular}

Table II NBT responses during episodes of infection

'False-negative' NBT scores 
following renal transplantation and in a recent report accounted for $39 \%$ of deaths in cadaver kidney recipients (Gurland et al, 1973). Diagnosis of the presence and site of infection is complicated by the use of immunosuppressive drugs which tend to mask clinical signs as well as interfering with helpful diagnostic features such as leucocytosis. The differentiation of infection from rejection is often difficult as some features such as pyrexia and deteriorating renal function can be common to both. It is recognized that delay in the diagnosis and hence the treatment of an acute rejection episode adversely affects the fate of the transplanted kidney. At present the diagnosis of acute rejection is made mainly on the basis of clinical signs such as pyrexia, weight gain, graft swelling and tenderness, and biochemical changes such as a falling urine volume and creatinine clearance. However, the biochemical changes are non-specific and are of no value in the early postoperative period before the graft has started to function. New diagnostic aids which have been advocated include techniques for studying the immunological process associated with rejection. Of these the leucocyte migration test shows greatest potential (Wood, Gray, Briggs, and Bell, 1973) while further evaluation of the rosette inhibition test has not confirmed its earlier promise (Wood and Gray, 1973). Other tests which have been advocated include measurement of urinary fibrinogen derivatives (Naish, Peters, and Shackman, 1973) and estimation of the enzyme $\mathrm{N}$-acetyl- $\beta \mathrm{D}$-glucosaminidase (Wellwood, Ellis, Hall, Robinson, and Thompson, 1973).

In the investigation of suspected infection, repeated culture of swabs, blood, urine, etc, is mandatory but a delay is inevitable before full reports are available and cultures are often negative, even in proven infection. In a series of 48 patients in our own unit, organisms were recovered in only 11 out of 16 bacterial infections. A rapid and reproducible test which would confirm the occurrence of infection and thus differentiate infection from rejection would, therefore, be of great value. The nitroblue tetrazolium test has been advocated as such a test (Wollman et al, 1972) while its value in the confirmation of systemic bacterial infection and its differentiation from non-infective syndromes has previously been demonstrated (Park et al, 1968; Lancet, 1971; Gordon et al, 1973). Wollman and his colleagues (1972) have studied NBT responses in uraemic patients and in renal transplant recipients during episodes of infection and rejection. The NBT test was shown to be strongly positive in transplant patients with bacterial infections but yielded normal test values during acute rejection episodes, thus permitting a differentiation between infection and rejection in these individuals. Our findings are in $\stackrel{0}{=}$ overall agreement with these observations. We have $\underline{\underline{\sigma}}$ noted enhanced NBT responses during six of the eight infective episodes encountered in this series. It has previously been reported that decreases in $\bar{C}$ NBT dye reduction are induced by immunosuppres- $\overline{\underline{G}}$ sive therapy thus producing false-negative NBT $\frac{\bar{\rho}}{\vec{\rho}}$ scores in the presence of systemic bacterial infection $\stackrel{\mathbb{Q}}{\Omega}$ (Miller and Kaplan, 1970; Matula and Paterson, § 1971). All of our patients were receiving prednisolone $\vec{\bullet}$ and azathioprine at the time of performing the NBT test. We have nevertheless shown that immuno- $\vec{\omega}$ suppressed renal transplant recipients remain capable $\stackrel{\Omega}{\rightarrow}$ of responding to bacterial infections with significant $\frac{8}{8}$ increases in the percentage of NBT-positive neutrophils, thus confirming the value of the technique in 6 the early detection of bacterial infection in such $\omega$ patients. One of our patients yielded NBT values $\stackrel{\omega}{+}$ within the normal range during each of two respira- $\frac{O}{3}$ tory infections, but these normal scores may be explained by the fact that they were clinically mild $\underset{\mathbb{D}}{\infty}$ infections unaccompanied by a pyrexia. Mild or $\frac{\mathbb{Q}}{\Phi}$ superficial non-systemic infection and concurrent antibiotic therapy are known causes of a diminished ${ }_{\mathbb{\Phi}}$ NBT response (Matula and Paterson, 1971; Rubinstein and Pelet, 1973; Gordon et al, 1973).

In our experience the NBT test was not influenced by episodes of transplant rejection. We obtaines NBT values within the normal range during each of the 11 rejections encountered in the present series despite the occurrence of pyrexia in several of the patients at some stage during the rejection. This confirms the findings of Wollman et al (1972) who $\vec{F}$ observed normal NBT scores during four rejection $\stackrel{\circ}{\exists}$ episodes in three patients. Although it has been shown that high serum steroid levels attained shortly after the administration of prednisolone may render neutrophils less responsive in the NBT test (Miller? and Kaplan; Wollman et al, 1972), the low NBT $\frac{5}{3}$ results encountered in our patients during their rejections cannot be explained on this basis since $\frac{\rho}{3}$ blood samples for the test were always collected before the intravenous administration of predniso- 9 lone. Segal, Trustey, and Levi (1973) have postu- $\rightarrow$ lated that low NBT scores recorded during transplant rejections may be due to 'a secondary abnormality $N$ of neutrophil function induced by circulating immune complexes'. There is as yet, no evidence to substantiate this hypothesis.

The NBT test was originally introduced as a screening procedure to identify patients with the $\mathrm{X}$-linked disorder chronic granulomatous disease. The test was also initially believed to distinguish $\stackrel{?}{+}$ clearly between bacterial and viral infections and $\underset{T}{0}$ pyrexial syndromes of non-infective origin (Park et al, 1968). Indeed, an editorial on this subject 
(Lancet, 1971) concluded 'its introduction into the repertoire of the routine hospital laboratory seems justified'. More recently an objective reassessment of the NBT test has rendered its status more doubtful (Segal et al, 1973; Steigbigel, Johnson, and Remington, 1974; Lancet, 1974). It would appear that the early promise shown by the test has not been fulfilled. There may be an overlap between normal and abnormal samples. Steigbigel et al (1974) have found routine haematological indices more sensitive than the NBT test as indicators of infection. Segal et al (1973) have found the technique relatively disappointing as a means of distinguishing between pyogenic infection and non-infective pyrexial disorders. These views are largely based on the interpretative difficulties which they considered might be encountered by inexperienced observers and the relatively poor results obtained with heparinized blood samples. Furthermore, numerous causes of false-positive and false-negative results in the NBT test have now been identified (Gordon et al, 1973; Lancet, 1974). However, certain specific clinical indications for using the NBT test remain. Its value for the detection of individuals with chronic granulomatous disease remains undisputed, and it has an important application in the early detection of bacterial infection in patients undergoing intensive care or those who are immunosuppressed (Freeman and King, 1972; Wollman et al, 1972; Winchester et al, 1973). The renal transplant recipient clearly belongs to the latter category. We believe that the NBT test can usefully be employed to differentiate between infection and rejection in such patients. In circumstances where this distinction is proving difficult, an elevated NBT response is good evidence in favour of infection.

References

Bell, P. R. F., Briggs, J. D., Kyle, K., Dick, H., Calman, K. C.,
Quin, R. O., Wood, R. F. M., Paton, A. M., Macpherson, S. G., Deane, R. F., McLaughlin, I., Hamilton, D. N. H., and Jackson, D. (1972). Renal transplantation: an analysis of 33 cases. Brit. med. J., 4, 408-413.

Briggs, J. D., Timbury, Morag C., Paton, A. M., and Bell, P. R. F. (1972). Viral infection and renal transplant rejection. Brit. med. J., 4, 520-522.

Freeman, R., and King, B. (1972). Infective complications of indwelling intravenous catheters and the monitoring of infections by the nitroblue-tetrazolium test. Lancet, 1, 992-993.

Gordon, A. M., Rowan, R. M., Brown, T., and Carson, H. G. (1973). Routine application of the nitroblue tetrazolium test in the clinical laboratory. J. clin. Path., 26, 52-56.

Gurland, H. J., Brunner, F. P., Dehn, H. v., Härlen, H., Parsons, F. M., and Schärer, K. (1973). Combined report on regular dialysis and transplantation in Europe, III, 1972. Proc. Europ. Dialysis Transplant Ass., 10, xvii-1 vi.

Lancet (1971). Editorial. Nitroblue tetrazolium: a routine test? Z, 909-910.

Lancet (1974). Editorial. Another look at the N.B.T. test. 1, 664-665.

Matula, G., and Paterson, P. Y. (1971). N.B.T. tests in a patient on steroids. Lancet, 1, 803-804.

Miller, D. R., and Kaplan, H. G. (1970). Decreased nitroblue tetrazolium dye reduction in the phagocytes of patients receiving prednisolone. Pediatrics, 45, 861-865.

Naish, P., Peters, D. K., and Shackman, R. (1973). Increased urinary fibrinogen derivatives after renal allotransplantation. Lancet, 1, 1280-1282.

Park, B. H., Fikrig, S. M., and Smithwick, E. M. (1968). Infection and nitroblue tetrazolium reduction by neutrophils: a diagnostic aid. Lancet, 2, 532-534.

Rubinstein, A., and Pelet, B. (1973). False-negative N.B.T. tests due to transient malfunction of neutrophils. Lancet, $1,382$.

Segal, A. W., Trustey, S. F., and Levi, A. J. (1973). Re-evaluation of nitroblue tetrazolium test. Lancet, 2, 879-883.

Steigbigel, R. T., Johnson, P. K., and Remington, J. S. (1974). The nitroblue tetrazolium reduction test versus conventional hematology in the diagnosis of bacterial infection. New Engl. J. Med., 290, 235-238.

Wellwood, J. M., Ellis, B. G., Hall, J. H., Robinson, D. R., and Thompson, A. E. (1973). Early warning of rejection. Brit. med. J., 2, 261-265.

Winchester, J. F., Gordon, A. M., Rowan, R. M., Lindsay, R. M., and Black, D. A. (1973). Interpretation of the nitroblue tetra. zolium test in regularly dialysed patients. Lancet, 2, 292-293.

Wollman, M. R., Brennan, B. L., Stenzel, K. H., David, D. S., Lewy, J. E., Rubin, A. L., and Miller, D. R. (1972). The nitrobluetetrazolium test. Lancet, 2, 289-291.

Wood, R. F. M., Gray, A. C., Briggs, J. D., and Bell, P. R. F. (1973). The prediction of acute rejection in human renal transplanta, tion using the leucocyte migration test. Transplantation, 1641-45.

Wood, R. F. M., and Gray, A. C. (1973). Evaluation of rosette intibition test in renal transplantation. Brit. med. J., 4, 649-65t. 